\title{
Uracil-tegafur and tamoxifen vs cyclophosphamide, methotrexate, fluorouracil, and tamoxifen in post-operative adjuvant therapy for stage I, II, or IIIA lymph node-positive breast cancer: a comparative study
}

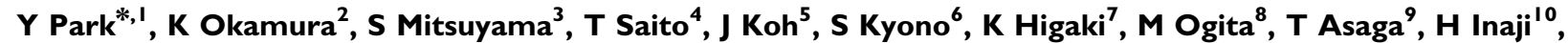 \\ H Komichi" ${ }^{11}$ N Kohno' ${ }^{12}$, K Yamazaki ${ }^{13}$, F Tanaka ${ }^{14}$, T Ito ${ }^{15}$, H Nishikawa ${ }^{16}$, A Osaki ${ }^{17}$, H Koyama ${ }^{10}$ and \\ T Suzuki ${ }^{10}$
}

'Department of Surgery, Toho University School of Medicine, Sakura Hospital, 564-I Shimoshizu, Sakura 285-874I, Japan; '2Department of Surgery, Kumamoto Rosai Hospital, 1670 Takehara-machi, Yatsushiro 866-8533, Japan; ${ }^{3}$ Department of Surgery, Kitakyushu Municipal Medical Center, 2-I - I Bashaku, Kokurakita-ku, Kitakyushu 802-0077, Japan; ${ }^{4}$ Department of Surgery, Omiya Red Cross Hospital, 8-3-33 Kamiochiai, Chuo-ku, Saitama 338-8553, Japan; ${ }^{5}$ Department of Surgery, Saitama Social Insurance Hospital, 4-9-3 Kitaurawa, Urawa-ku, Saitama 330-0074, Japan; ' Department of Surgery, Nippon Medical School, Chiba-hokuso Hospital, 1715 Kamagari, Inba-mura, Inba-gun, Chiba 270-1694, Japan; ${ }^{7}$ Department of Surgery, Hiroshima Municipal Hiroshima Hospital, 7-33 Motoi-machi, Naka-ku, Hiroshima 730-85 I 8, Japan; ${ }^{8}$ Division of Mammary Endocrinology, Department of Surgery, National Sapporo Hospital, 4-2 Kikusui, Shiroishi-ku, Sapporo 003-0804, Japan; 'Department of Breast and Thyroid Surgery, Kanagawa Cancer Center Hospital, I-I-2 Nakao, Asahi-ku, Yokohama 24I-0815, Japan; ${ }^{10}$ Department of Surgery, Osaka Medical Center for Cancer and Cardiovascular Diseases, I-3-3 Nakamichi, Higashinari-ku, Osaka 537-85 I I, Japan; " Department of Surgery, National Nara Hospital, 2-789 Shichijo, Nara 630-8053, Japan; ${ }^{12}$ Department of Surgery, Hyogo Medical Center for Adult Disease, 13-70 Kitaoji-cho, Akashi 673-8558, Japan; ${ }^{13}$ Department of Surgery, Asahikawa Medical College, 2-I I I Midorigaoka-Higashi, Asahikawa 078-85 I 0, Japan; ${ }^{4}$ Department of Surgery, Fukui Red Cross Hospital, 2-4-I Tukimi, Fukui 918-850 I, Japan; ${ }^{15}$ Department of Breast Surgery, Gifu Municipal Hospital, 7-I Kashima-cho, Gifu 500-85 I 3, Japan;

${ }^{16}$ Department of Surgery, Hyogo Prefectural Amagasaki Hospital, I-I-I Higashidaimotsu-cho, Amagasaki 660-0828, Japan; ${ }^{17}$ Department of Surgery, Research Institute for Radiation Biology and Medicine, Hiroshima University, I-2-3 Kasumi, Minami-ku, Hiroshima 734-8553, Japan

BACKGROUND: It has been reported that treatment with uracil-tegafur (UFT) has shown significantly better survival and relapse-free survival (RFS) than surgery alone. Therefore, we compared UFT with a combination therapy of cyclophosphamide, methotrexate, and fluorouracil (CMF) in patients who had undergone curative surgery for axillary lymph node-positive breast cancer.

METHODS: A total of 377 node-positive patients with stage I, II, or IIIA disease were registered from September 1996 through July 2000 and were randomly assigned to either 6 cycles of CMF or 2 years of UFT. In both arms, tamoxifen (TAM) was concurrently administered for 2 years. The primary end point in this study was the non-inferiority of UFT to CMF.

RESULTS: No statistically significant difference between the two groups was observed with regard to the 5-year RFS rate (72.2\% in the UFT and $76.3 \%$ in the CMF). Adverse event profiles differed between the two groups, with a significantly lower incidence of leukopenia and anaemia in the UFT group, as well as anorexia, nausea/vomiting, stomatitis, and alopecia, which have implications for quality of life.

CONCLUSION: UFT administered in combination with TAM holds promise in the treatment of lymph node-positive early breast cancer. On stratified analysis, the recurrence rate in the UFT group was found to be better in oestrogen receptor (ER)-positive patients. Tegafur-based treatment should be evaluated by a prospective randomised trial conducted in ER-positive patients.

British Journal of Cancer (2009) I 0I, 598-604. doi:I0.1038/sj.bjc.66052I 8 www.bjcancer.com

Published online 28 July 2009

(c) 2009 Cancer Research UK

Keywords: breast cancer; combination therapy; CMF; tamoxifen; randomised trial; uracil-tegafur

The Early Breast Cancer Trialists' Collaborative Group (EBCTCG) conducts a meta-analysis of randomised studies of post-operative adjuvant therapy for breast cancer every 5 years (EBCTCG, 2005). They reported that polychemotherapy (cyclophosphamide (CPA),

* Correspondence: Dr Y Park, Department of Surgery, Toho University School of Medicine, Sakura Hospital, 564-I Shimoshizu, Sakura, Chiba 285-874I, Japan; E-mail: youngjinpark@sakura.med.toho-u.ac.jp

Received 30 March 2009; revised 30 June 2009; accepted 3 July 2009; published online 28 July 2009 methotrexate (MTX), fluorouracil (5-FU) ((CMF))-based; anthracycline-based; or other types of polychemotherapy) was associated with a decrease in the odds ratio of disease-free survival and an overall improvement in survival compared with no chemotherapy (EBCTCG, 1998).

The relatively recent addition of taxanes to anthracycline-based chemotherapy has markedly improved outcomes of post-operative chemotherapy for node-positive breast cancer (Ferguson et al, 2009). Furthermore, concomitant chemotherapy with trastuzumab, an antihuman epidermal growth factor receptor 2 (HER2) antibody, has been reported to substantially improve outcomes in resected HER2-positive 
breast cancer (Mackey et al, 2009). Selection of the most suitable drugs and regimens should be done in careful consideration of anticipated risks, expected benefits, and estimated costs.

Associated with fewer adverse events such as cardiotoxicity and bone marrow suppression compared with anthracycline- or taxane-based regimens, CMF therapy remains widely used as a therapeutic option. Furthermore, if therapeutic drugs could be administered orally instead of being intravenously infused, patients with breast cancer would benefit greatly because oral formulations are more convenient, are associated with fewer adverse events such as alopecia, and have higher compliance.

With regard to monotherapy regimens, oral fluoropyrimidines such as uracil-tegafur (UFT) (Taguchi, 1997), which are suitable for ambulatory-based treatment, have been used in Japan since the 1980s. Tashiro et al (1994) reported a response rate for UFT in patients with recurrent breast cancer of $39 \%$, which is comparable with the $36 \%$ reported for CMF in patients with advanced or recurrent breast cancer in Japan (Nomura et al, 1994), as well as markedly lower toxicity for UFT than CMF. These results suggest that UFT likely prevents relapse as effectively as CMF in postoperative adjuvant therapy for breast cancer, but with a lower severity of adverse events.

Furthermore, in the adjuvant setting, treatment with UFT has shown significantly better survival and relapse-free survival (RFS) than surgery alone in prospective randomised clinical trials in breast cancer (Kasumi et al, 2003; Noguchi et al, 2005).

Here, we conducted a randomised study to compare UFT with CMF in patients with axillary lymph node-positive stage I, II, or IIIA breast cancer.

\section{PATIENTS AND METHODS}

\section{Eligibility criteria}

Eligibility criteria included (1) TNM stage I, II, or IIIA; (2) involvement of $1-9$ axillary lymph nodes; (3) curative surgery; (4) age 20-65 years; (5) body weight $\geqslant 40 \mathrm{~kg}$; (6) normal haematological parameters as well as adequate hepatic and renal function; and (7) written informed consent. Patients with bilateral breast cancer, male breast cancer, inflammatory breast cancer, and double cancers were excluded, as were pregnant or lactating women with breast cancer. Patients were eligible regardless of their oestrogen receptor (ER) status. The cutoff level of ER was $13 \mathrm{fmol} \mathrm{mg}{ }^{-1}$ soluble fraction in terms of enzyme immunoassay. Consecutive patients who met all of the eligibility criteria and received treatment as specified were included in the analysis.

\section{Study design}

The study scheme is shown in Figure 1. The UFT (Taiho Pharmaceuticals Co. Ltd., Tokyo, Japan) group received tamoxifen (TAM, AstraZeneca K.K., Osaka, Japan) $\left(20 \mathrm{mg} \mathrm{day}^{-1}\right)$ in combination with UFT $\left(270 \mathrm{mg} \mathrm{m}^{-2} \mathrm{day}^{-1}\right)$ for 2 years. The CMF group received TAM $\left(20 \mathrm{mg} \mathrm{day}^{-1}\right)$ for 2 years in combination with 6 cycles of CPA (Shionogi \& Co. Ltd., Osaka, Japan) $\left(65 \mathrm{mg} \mathrm{m}^{-2}\right.$ : days $1-14)$, MTX (Wyeth K.K., Tokyo, Japan) $\left(40 \mathrm{mg} \mathrm{m}^{-2}\right.$ : days 1 and 8), and 5-FU (Kyowa Hakko Kirin Co. Ltd., Tokyo, Japan) $\left(500 \mathrm{mg} \mathrm{m}^{-2}\right.$ : days 1 and 8$)$, with the cycle repeated every 4 weeks.

Laboratory tests and observation of signs and symptoms were carried out every month in the UFT group for the first 6 months and thereafter at intervals of 3 months for the remainder of the 2 -year treatment period, and on days 1,8 , and 15 of each cycle in the CMF group. Adverse events were assessed according to the grade classification of the Japan Society of Clinical Oncology, which are equivalent to the World Health Organization in all substantial aspects. Eligibility of subjects, cases of relapse, and deaths were clinically reviewed by the Data Review Committee. The study protocol was approved by the institutional review board of each participating institution. All procedures were carried out in accordance with the Declaration of Helsinki.

\section{Statistical considerations}

The primary end point in this study was the non-inferiority of the UFT group to the CMF group, as measured by the RFS rate. The secondary end point was superiority with regard to a lower

Mastectomy

Central randomisation

Minimisation: $\mathrm{T}$ factor (T1, T2, T3), $\mathrm{n}$ factor (1-3, 4-6, 7-9)

Age $(-49,50-$ years), ER (+, -, unknown)

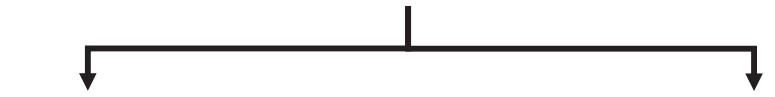

CMF+TAM

UFT+TAM

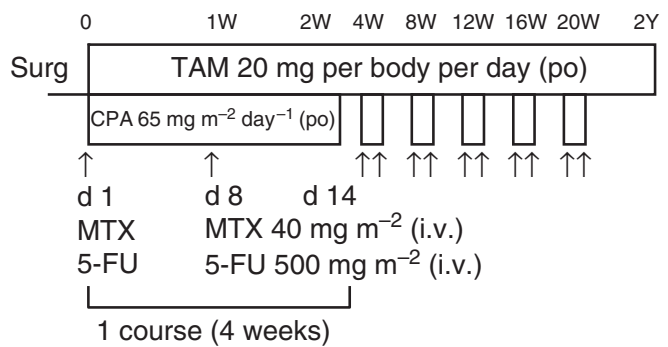

\begin{tabular}{c|c|}
\multicolumn{1}{c}{\begin{tabular}{c}
0 \\
\cline { 2 - 2 } Surg
\end{tabular}} & TAM $20 \mathrm{mg}$ per body per day (po) \\
\hline & UFT $270 \mathrm{mg} \mathrm{m}^{-2}$ day $^{-1}$ (po) \\
\cline { 2 - 2 }
\end{tabular}

Follow-up: every 3 months for 2 years and every 6 months thereafter

Patient enrolment: September 1996-July 2000

Figure I Study design. ER, oestrogen receptor; CMF, cyclophosphamide-methotrexate-fluorouracil; TAM, tamoxifen; UFT, uracil-tegafur; W, week; Y, year; Surg, surgery; po, per os; CPA, cyclophosphamide; d, day; MTX, methotrexate; 5-FU, fluorouracil; i.v., intravenous. 
incidence of adverse events. Given that the EBCTCG study reported a $9.4 \pm 1.4 \%$ difference in 5 -year recurrence-free survival rate in node-positive patients between the CMF therapy and control groups (EBCTCG, 1992), we adopted an approximate value of $\Delta 10 \%$ to demonstrate clinical non-inferiority. We estimated a 5 -year RFS rate of $70 \%$ in both the UFT and the CMF groups. As $\Delta 10 \%$ corresponds to a hazard ratio (HR) of 1.43 , demonstration of non-inferiority required the upper limit of the $90 \%$ confidence interval for the HR of the UFT group relative to the CMF group to be $\leqslant 1.43$. Demonstration of non-inferiority at a significance level of 0.05 (one-sided) with a power of $80 \%$ in turn required a sample size of 648 subjects. Therefore, we selected a sample size of 680 subjects, in consideration of the likely number of ineligible subjects and subjects not treated as specified.

Distribution of background factors and the incidence of adverse events were analysed using the $\chi^{2}$ test and Mann - Whitney $U$-test, respectively. Relapse-free survival time was defined as the period from the day of surgery to the day of final confirmation of a relapse-free status or to the first confirmation of relapse. For subjects who died of any cause other than relapse of the underlying disease, RFS time was defined as the period from the day of surgery to the day of death, with death considered as an event. Survival time was defined as the period from the day of surgery to the day of final confirmation of survival or to the confirmation of death from any cause.

Analyses in this study were performed on evaluable patients. Analyses were carried out using SAS software (version 6.12, SAS Institute, Cary, NC, USA), with the PHREG procedure used to estimate HR. In accordance with changes introduced by the ICH International Conference on Harmonization E9 (1998), the upper limit of the confidence intervals was changed to $95 \%$. Both RFS and survival were estimated using the Kaplan-Meier method and analysed by the log-rank test. A $P$-value $<0.05$ was considered to indicate a statistically significant difference.

\section{RESULTS}

\section{Enrolment and patient characteristics}

Patient characteristics are shown in Table 1. Recruitment began in September 1996 at 100 participating clinical sites in Japan. The recruitment period was extended in June 1988 from the originally planned 2 years to 4 years because the target number of subjects had not been reached. Despite this, only half the target sample size was achieved because of slow accrual and, as a result, recruitment was terminated in July 2000 with 377 enrolled patients. Among the 377 patients, 188 were randomised to the UFT group, with 3 excluded as being ineligible and 8 for protocol violations, leaving 177 patients for inclusion in the analysis. For the CMF group, 189 patients were randomised, with 7 excluded as being ineligible and 9 because of protocol violations, leaving 173 patients for analysis. No marked bias was observed in age distribution, hormone receptor status, number of involved axillary lymph nodes, or histological type. The frequency of ER-positive cases in the UFT and CMF groups was the same (51.4\%).

\section{Efficacy}

Both RFS and survival curves are shown in Figure 2. The 5-year RFS rates in the UFT and CMF groups were 72.2 and $76.3 \%$, respectively, with no statistically significant difference between them (log-rank test, $P=0.46$ ). The $\mathrm{HR}$ for the UFT group relative to the CMF group in terms of RFS rate was $1.18(95 \%$ CI: $0.77-1.80$ ). Five-year survival rates in the UFT and CMF groups were 87.0 and $88.4 \%$, respectively, showing no statistically significant difference between them, and the HR was 1.15 (log-rank test, $P=0.66)$. Subset analysis showed that there were non-
Table I Patient characteristics

\begin{tabular}{|c|c|c|}
\hline & CMF & UFT \\
\hline $\begin{array}{l}\text { No. of enrolled patients } \\
\text { No. of eligible patients } \\
\text { No. of evaluable patients }\end{array}$ & $\begin{array}{l}189 \\
182 \\
173\end{array}$ & $\begin{array}{l}188 \\
185 \\
177\end{array}$ \\
\hline $\begin{array}{l}\text { Age (mean } \pm \text { s.d.) } \\
\quad<50 \text { years } \\
\geqslant 50 \text { years }\end{array}$ & $\begin{array}{c}51.0 \pm 7.7 \\
88 \\
85\end{array}$ & $\begin{array}{c}51.7 \pm 7.8 \\
88 \\
89\end{array}$ \\
\hline Body surface area $\left(m^{2}\right.$, mean \pm s.d. $)$ & $1.47 \pm 0.11$ & $1.48 \pm 0.11$ \\
\hline $\begin{array}{l}T^{T} \\
\text { T1 } \\
\text { T2 } \\
\text { T3 }\end{array}$ & $\begin{array}{l}36 \\
122 \\
15\end{array}$ & $\begin{array}{c}31 \\
132 \\
14\end{array}$ \\
\hline $\begin{array}{l}1-3 \\
4-6 \\
7-9\end{array}$ & $\begin{array}{l}135 \\
26 \\
12\end{array}$ & $\begin{array}{l}138 \\
25 \\
14\end{array}$ \\
\hline $\begin{array}{l}\text { Hormone receptor status } \\
\operatorname{ER}(-) \text { and } \operatorname{PgR}(-) \\
\text { Others }\end{array}$ & $\begin{array}{l}52 \\
121\end{array}$ & $\begin{array}{c}53 \\
124\end{array}$ \\
\hline $\begin{array}{l}\text { ER }(-) \\
\text { ER(+) } \\
\text { Unknown }\end{array}$ & $\begin{array}{l}73 \\
89 \\
11\end{array}$ & $\begin{array}{l}72 \\
91 \\
14\end{array}$ \\
\hline $\begin{array}{l}\operatorname{PgR}(-) \\
\operatorname{PgR}(+) \\
\text { Unknown }\end{array}$ & $\begin{array}{c}73 \\
78 \\
8\end{array}$ & $\begin{array}{c}73 \\
78 \\
6\end{array}$ \\
\hline $\begin{array}{l}\text { Histological pattern } \\
\text { Invasive ductal ca. } \\
\text { Lobular ca. } \\
\text { Others }\end{array}$ & $\begin{array}{l}165 \\
5 \\
3\end{array}$ & $\begin{array}{l}160 \\
7 \\
10\end{array}$ \\
\hline
\end{tabular}

$\mathrm{ca} .=$ carcinoma; $\mathrm{CMF}=$ cyclophosphamide - methotrexate-fluorouracil; $E \mathrm{R}=$ oestrogen receptor; $\mathrm{PgR}=$ progesterone receptor, UFT = uracil-tegafur.

significant tendencies of interaction between RFS and age, number of involved lymph nodes and ER status (Figure 3).

The number of cases of relapse and the distribution of sites are shown in Table 2. There were $45(25.4 \%)$ and $39(22.5 \%)$ cases of relapse in the UFT and CMF groups, respectively. The most common initial site of relapse was soft tissue, followed by bone and visceral organs in both the groups. In addition, neither secondary breast cancer nor other malignancies were observed in both the groups. No difference in the number of cases or distribution of sites was observed between the two groups.

\section{Safety}

Adverse events are shown in Table 3. Adverse events were reported in $88.1 \%$ (156 out of 177 ) of patients receiving UFT and in $98.8 \%$ (171 out of 173) of those receiving CMF, showing a significantly lower incidence in the UFT group $(P<0.05)$. The incidence of leukopenia as well as haemoglobin, anorexia, nausea/vomiting, stomatitis, and alopecia was significantly higher in the CMF group, whereas that of liver dysfunction was significantly higher in the UFT group.

\section{Dose and concomitant medications}

The mean total dose of UFT was $75.2 \%$ of the target dose, whereas the mean total doses of CPA, MTX, and 5-FU were 85.8, 83.3, and $83.7 \%$ of the target dose, respectively. The mean total dose of TAM in the UFT and CMF groups was 85.7 and $86.7 \%$ of the target dose, respectively, and thus was not different between the two groups. 


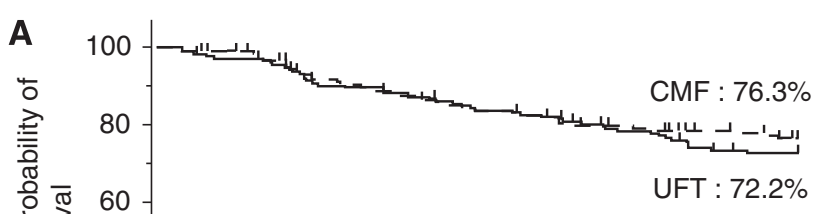
95\% Cl: 0.77-1.80
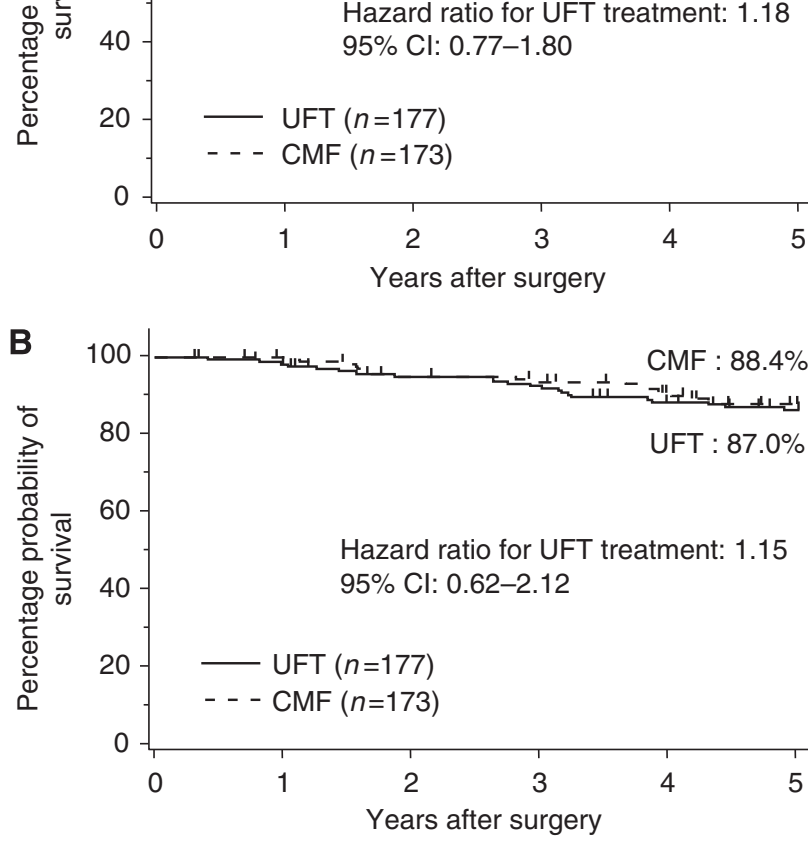

Figure 2 Relapse-free and overall survival. (A) Relapse-free survival; (B) overall survival. CMF, cyclophosphamide-methotrexate-fluorouracil; UFT, uracil-tegafur; $\mathrm{Cl}$, confidence interval.
Granulocyte colony-stimulating factors (G-CSFs) were used in 10 subjects (5.8\%) in the CMF group, but not in any subjects in the UFT group. We used 5-hydroxytryptamine $3\left(5-\mathrm{HT}_{3}\right)$ antagonists in 80 subjects $(46.2 \%)$ in the CMF group, but in only 1 subject $(0.6 \%)$ in the UFT group. The use of both G-CSFs and anti-emetics was statistically significantly less frequent in the UFT group (both $P=0.001$ )

\section{CONCLUSION}

In this study, we found no statistically significant difference in RFS among patients who had undergone curative surgery for axillary lymph node-positive breast cancer between those receiving UFT and CMF, at 72.2 vs $76.3 \%$, respectively. In addition, the UFT

Table 2 Sites of relapse

\begin{tabular}{lcc}
\hline & CMF & UFT \\
\hline No. of evaluable patients & 173 & 177 \\
No. of relapse cases & $39(22.5 \%)$ & $45(25.4 \%)$ \\
Soft tissue & $18(10.4 \%)$ & $23(13.0 \%)$ \\
Skin and subcutaneous tissues & $9(5.2 \%)$ & $12(6.8 \%)$ \\
Lymph node & $10(5.8 \%)$ & $12(6.8 \%)$ \\
Contralateral breast & $1(0.6 \%)$ & \\
Bone & $15(8.7 \%)$ & $12(6.8 \%)$ \\
& $14(8.1 \%)$ & $19(10.7 \%)$ \\
Visceral organs & $8(4.6 \%)$ & $12(6.8 \%)$ \\
Lung and pleura & $7(4.0 \%)$ & $7(4.0 \%)$ \\
Liver & $2(1.2 \%)$ & $2(1.1 \%)$ \\
Brain & $1(0.6 \%)$ & \\
Others & & \\
\hline
\end{tabular}

$\mathrm{CMF}=$ cyclophosphamide-methotrexate-fluorouracil; UFT = uracil-tegafur.

\section{Hazard ratio}

$(95 \% \mathrm{Cl})$

All patients

$1.18(0.80-1.80)$

Age, years

$<50$

$\geqslant 50$

Nodal status

n1-3

n4-9

ER

Negative

Positive

$\mathrm{PgR}$

Negative

Positive

$\mathrm{ER} / \mathrm{PgR}$

Both negative

ER-positive or PgR-positive
No. of patients

350
160
190

273
77

145
180

146
156

105
206

Risk reduction

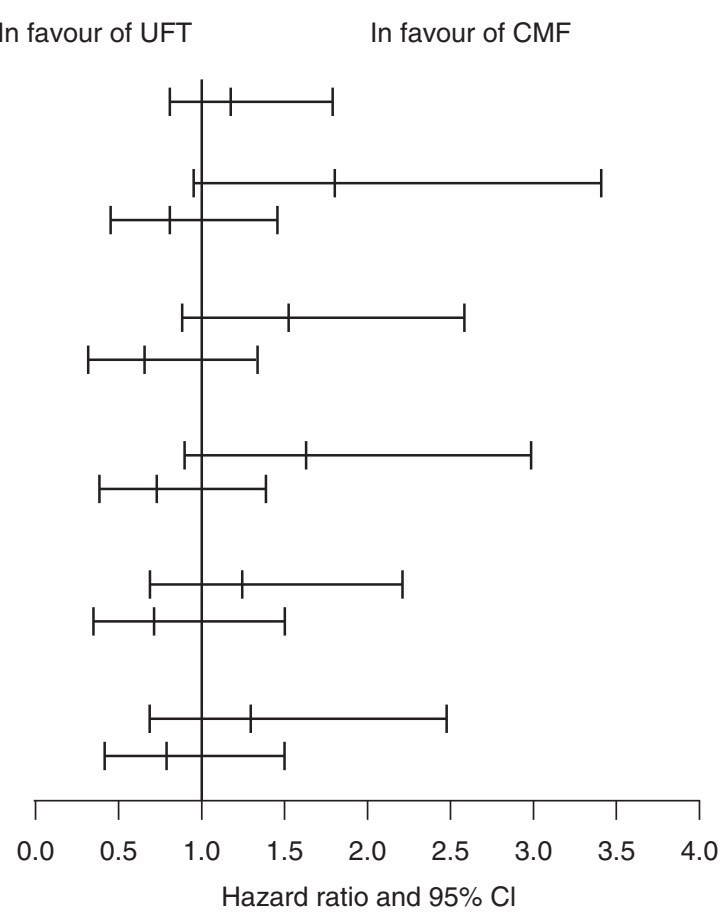

Test for interaction

0.07

0.26

0.28

Figure 3 Relapse-free survival by age, number of involved nodes and $E R, P g R$, and ER/PgR status. 
Table 3 Adverse events

CMF $(n=173)$

\begin{tabular}{|c|c|c|c|c|c|c|c|c|c|c|c|c|c|}
\hline & \multicolumn{5}{|c|}{ Grade } & \multirow[b]{2}{*}{ Incidence (\%) } & \multicolumn{5}{|c|}{ Grade } & \multirow[b]{2}{*}{ Incidence (\%) } & \multirow[b]{2}{*}{$P$-value } \\
\hline & Unknown & I & 2 & 3 & 4 & & Unknown & I & 2 & 3 & 4 & & \\
\hline Leukopenia & & 87 & 50 & 6 & & 82.7 & & 49 & 10 & & & 33.3 & $P<0.001$ \\
\hline Thrombocytopenia & & 21 & 2 & & & 13.3 & & 35 & 2 & & & 20.9 & \\
\hline Haemoglobin & & 52 & 2 & 1 & & 31.8 & & | | & 2 & & & 7.3 & $P<0.001$ \\
\hline AST & & 45 & | | & & & 32.4 & & 45 & 16 & & । & 35.0 & \\
\hline $\mathrm{ALT}$ & & 47 & 16 & & & 36.4 & & 51 & 16 & 2 & & 39.0 & \\
\hline Hepaplastin decrease & & । & & & & 0.6 & & 9 & & & & 5.1 & $P<0.05$ \\
\hline Total bilirubin & & 3 & & & & 1.7 & & 43 & 3 & । & & 26.6 & $P<0.001$ \\
\hline ALP & & 4 & & & & 2.3 & & 25 & & & & $\mid 4.1$ & $P<0.001$ \\
\hline BUN & & 2 & 1 & & & 1.7 & & 10 & & & & 5.6 & \\
\hline Creatinine & & & & & & - & & | & & & & 0.6 & \\
\hline Anorexia & & 62 & 18 & 6 & & 49.7 & I & 34 & 4 & । & & 22.6 & $P<0.001$ \\
\hline Nausea/vomiting & & 84 & 28 & 8 & & 69.4 & I & 32 & 6 & । & & 22.6 & $P<0.001$ \\
\hline Diarrhoea & I & 8 & & & & 5.2 & 2 & $\mid 1$ & & & & 7.3 & \\
\hline Stomatitis & & 17 & 3 & & & 11.6 & & 2 & 3 & & & 2.8 & $P=0.002$ \\
\hline Pigmentation & & 7 & 2 & & & 5.2 & & 12 & 4 & & & 9.0 & \\
\hline Numbness in fingers & & 1 & & & & 0.6 & & & I & & & 0.6 & \\
\hline Alopecia & & 57 & | | & & & 9.3 & & 4 & & & & 2.3 & $P<0.001$ \\
\hline
\end{tabular}

${ }^{a} \chi^{2}$ test. $A L P=$ alkaline phosphatase; $A L T=$ alanine aminotransferase; $A S T=$ aspartate aminotransferase; $B U N=$ blood urea nitrogen; $C M F=$ cyclophosphamide methotrexate-fluorouracil; UFT = uracil-tegafur.

group had significantly lower rates of adverse events compared with the CMF group $(P<0.05)$. Moreover, G-CSFs were used by 0 and $5.8 \%$ of subjects who received UFT and CMF therapies, respectively, and $5-\mathrm{HT}_{3}$ antagonists by 0.6 and $46.2 \%$, respectively.

One particular limitation of this study warrants mention. Owing to its small sample size, this study did not have sufficient statistical power to demonstrate the non-inferiority of UFT to CMF. However, the HRs for RFS and survival calculated over 5 years were 1.18 and 1.15 , respectively, which are consistent with the RFS and survival curves. Furthermore, the number of cases was sufficient enough to verify significant differences between the UFT and CMF groups with regard to adverse events. The UFT group had a significantly lower incidence of adverse events. These differences indicate the possibility of using UFT as a treatment option in place of CMF.

Similar findings were recently reported in a randomised study of stage I-IIIA node-negative, pathologically high-risk breast cancer, which compared a 2-year administration of UFT with 6 cycles of CMF (Watanabe et al, 2009). Results indicated that the efficacy of UFT as an adjuvant treatment was comparable with that of CMF. On the other hand, Cancer and Leukemia Group B (CALGB) reported that a 4.5-month administration of capecitabine was inferior to $\mathrm{CMF} / \mathrm{AC}$ (doxorubicin $(\mathrm{ADM})+\mathrm{CPA}$ ) in elderly breast cancer patients (Muss et al, 2009). This disagreement among results, including those of this study, might be partly explained by the difference in the duration of treatment with oral fluoropyrimidines.

An additional finding of our study was that subset analysis indicated a tendency of interaction among age, number of involved lymph nodes, or ER status and RFS, suggesting that these subsets may affect therapeutic effect. Among these three subsets, it is unclear why UFT was superior to CMF in patients with four or more metastatic axillary lymph nodes. With regard to age in premenopausal women, relapse prevention by CMF is mainly attributable to its chemical oophorectomy effect, but in post-menopausal women primarily to other mechanisms, such as cytotoxic effects.

In contrast, UFT is reported to have anti-angiogenic effects in addition to its cytotoxic effects based on the evaluation in BALB/c mouse models (Yonekura et al, 1999; Munoz et al, 2006). These findings may explain the higher efficacy of UFT than CMF in patients aged $\geqslant 50$ years. With regard to ER status, our findings support previous findings that concurrent treatment with UFT and
TAM yields better outcomes in patients with ER-positive breast cancer than does monotherapy with TAM (Noguchi et al, 2005). Given the finding by Albain (1989) that the CAF-TAM combination treatment is more effective in sequential than in concurrent administration, combination treatment with anthracycline-based infusional therapy with TAM is believed to have equivalent or lower efficacy when administered concurrently than sequentially. However, the validity of the concurrent approach in vitro has been demonstrated (Kurebayashi et al, 2007). The combination of 4-hydroxy-TAM (4OHT) and 5-FU had an additive effect in inhibiting the proliferation of ER- $\alpha$-positive cells, whereas that of $4 \mathrm{OHT}$ and ADM worked antagonistically. With regard to changes in gene expression for susceptibility or tolerance to TAM, 5-FU, or ADM, 5-FU did not change the expression level of TAM-related genes, whereas 4OHT significantly inhibited thymidylase synthase, which is a key enzyme in the antitumour activity of 5-FU, and thereby enhanced the anti-tumour effect of 5-FU. In contrast, ADM did not change gene expression for TAM susceptibility, but did increase the expression of some genes for TAM tolerance. The authors considered this to indicate the fact that ADM in combination with $4 \mathrm{OHT}$ may exert a rather negative anti-tumour effect. Furthermore, anthracyclines are reportedly less effective against HER2-negative than HER2-positive breast cancers (Gennari et al, 2008), whereas the relapsepreventative effect of UFT is not affected by HER2 status (Toi et al, 2007).

The Consensus Guideline (Goldhirsch et al, 2007) established at the St Gallen Conference states that no therapeutic modality has been established for HER2-negative and hormone receptor-positive intermediate-risk breast cancer. The question of whether postoperative endocrine therapy alone is sufficient in these patients or should be supplemented with chemotherapy remains unanswered. As mentioned above, as UFT can prevent ER-positive breast cancer from recurring when combined with hormone therapy, regardless of HER2 status, it may provide a useful therapeutic option for patients with intermediate-risk breast cancer.

In conclusion, these findings suggest that UFT may hold promise as a treatment for the prevention of relapse of nodepositive early breast cancer. Tegafur-based treatment should be evaluated by a prospective randomised trial conducted in ER-positive patients. 


\section{ACKNOWLEDGEMENTS}

The following investigators are members of the Data Review Committee: Hideo Inaji, Yuichi Takatsuka, Hiroji Iwata, Hideya Tashiro, Eisei Shin, Takaichiro Suzuki, and Kojiro Shimozuma. This study was presented in part at the 40th Annual Meeting of the

\section{REFERENCES}

Albain KS (1989) Adjuvant chemotherapy and endocrine therapy for nodepositive and node-negative breast carcinoma. Clin Obstet Gynecol 32: $835-857$

Early Breast Cancer Trialist's Collaborative Group (1992) Systemic treatment of early breast cancer by hormonal, cytotoxic, or immune therapy. 133 randomised trials involving 31,000 recurrences and 24,000 deaths among 75,000 women. Early Breast Cancer Trialists' Collaborative Group. Lancet 339: 71-85

Early Breast Cancer Trialists' Collaborative Group (1998) Polychemotherapy for early breast cancer: an overview of the randomised trials. Early Breast Cancer Trialists' Collaborative Group. Lancet 352: $930-942$

Early Breast Cancer Trialists' Collaborative Group (EBCTCG) (2005) Effects of chemotherapy and hormonal therapy for early breast cancer on recurrence and 15-year survival: an overview of the randomised trials. Lancet 365: 1687-1717

Ferguson T, Wilcken N, Vagg R, Ghersi D, Nowak AK (2009) Taxanes for adjuvant treatment of early breast cancer. Cochrane Database Systematic Review. Issue 2 Art No.: CD004421 pub2

Gennari A, Sormani MP, Pronzato P, Puntoni M, Colozza M, Pfeffer U, Bruzzi P (2008) HER2 status and efficacy of adjuvant anthracyclines in early breast cancer: a pooled analysis of randomized trials. J Natl Cancer Inst 100: $14-20$

Goldhirsch A, Wood WC, Gelber RD, Coates AS, Thurlimann B, Senn HJ (2007) Progress and promise: highlights of the international expert consensus on the primary therapy of early breast cancer. 2007. Ann Oncol 18: $1133-1144$

Kasumi F, Yoshimoto M, Uchino J, Abe R, Nomura Y, Sugimachi K, Nakazato H, Abe O (2003) Meta-analysis of five studies on tegafur plus uracil (UFT) as post-operative adjuvant chemotherapy for breast cancer. Oncology 64: 146-153

Kurebayashi J, Nukatsuka M, Nagase H, Nomura T, Hirono M, Yamamoto Y, Sugimoto Y, Oka T, Sonoo H (2007) Additive antitumor effect of concurrent treatment of 4-hydroxy tamoxifen with 5-fluorouracil but not with doxorubicin in estrogen receptor-positive breast cancer cells. Cancer Chemother Pharmacol 59: 515-525

Mackey J, McLeod D, Ragaz J, Gelmon K, Verma S, Pritchard K, Laing K, Provencher L, Charbonneau LF (2009) Adjuvant targeted therapy in early breast cancer. Cancer 115: 1154-1168
American Society of Clinical Oncology, 5-8 June 2004, New Orleans, LA, USA.

The authors are indebted to Professor J Patrick Barron of the International Medical Communications Center of Tokyo Medical University, a paid consultant of Taiho Pharmaceutical Co. Ltd, for his review of this manuscript.

Munoz R, Man S, Shaked Y, Lee CR, Wong J, Francia G, Kerbel RS (2006) Highly efficacious nontoxic preclinical treatment for advanced metastatic breast cancer using combination oral UFT-cyclophosphamide metronomic chemotherapy. Cancer Res 66: 3386-3391

Muss HB, Berry DA, Cirrincione CT, Theodoulou M, Mauer AM, Kornblith AB, Partridge AH, Dressler LG, Cohen HJ, Becker HP, Kartcheske PA, Wheeler JD, Perez EA, Wolff AC, Gralow JR, Burstein HJ, Mahmood AA, Magrinat G, Parker BA, Hart RD, Grenier D, Norton L, Hudis CA, Winer EP, CALGB Investigators (2009) Adjuvant chemotherapy in older women with early-stage breast cancer. $N$ Engl J Med 360: 2055-2065

Noguchi S, Koyama H, Uchino J, Abe R, Miura S, Sugimachi K, Akazawa K, Abe O (2005) Postoperative adjuvant therapy with tamoxifen, tegafur plus uracil, or both in women with node-negative breast cancer: a pooled analysis of six randomized controlled trials. J Clin Oncol 23: 2172-2184

Nomura Y, Tominaga T, Adachi I, Koyama H, Fukami A (1994) [Clinical evaluation of cyclophosphamide, methotrexate and 5-fluorouracil (CMF) on advanced and recurrent breast cancer. Clinical study group of CMF for breast cancer in Japan]. Gan To Kagaku Ryoho 21: 1949-1956

Taguchi T (1997) Clinical application of biochemical modulation in cancer chemotherapy: biochemical modulation for 5-FU. Oncology 54(Suppl 1): $12-18$

Tashiro H, Nomura Y, Ohsaki A (1994) A double blind comparative study of tegafur (FT) and UFT (a combination of Tegafur and uracil) in advanced breast cancer. Jpn J Clin Oncol 24: 212-217

Toi M, Ikeda T, Akiyama F, Kurosumi M, Tsuda H, Sakamoto G, Abe O (2007) Predictive implications of nucleoside metabolizing enzymes in premenopausal women with node-positive primary breast cancer who were randomly assigned to receive tamoxifen alone or tamoxifen plus tegafur-uracil as adjuvant therapy. Int J Oncol 31: 899-906

Watanabe T, Sano M, Takashima S, Kitaya T, Tokuda Y, Yoshimoto M, Kono N, Nakagami K, Iwata H, Shimozuma K, Sonoo H, Tsuda H, Sakamoto G, Ohashi Y (2009) Oral uracil and tegafur compared with classic cyclophosphamide, methotrexate, fluorouracil as postoperative chemotherapy in patients with node-negative, high-risk breast cancer: National Surgical Adjuvant Study for Breast Cancer 01 trial. J Clin Oncol 27: 1368-1374

Yonekura K, Basaki Y, Chikahisa L, Okabe S, Hashimoto A, Miyadera K, Wierzba K, Yamada Y (1999) UFT and its metabolites inhibit the angiogenesis induced by murine renal cell carcinoma, as determined by a dorsal air sac assay in mice. Clin Cancer Res 5: 2185-2191

\section{Appendix}

\section{Participating institutions}

\begin{tabular}{lc}
\hline Asahikawa Medical College & Toho University School of Medicine, Sakura Hospital \\
Kushiro Rosai Hospital & Nippon Medical School, Chiba-hokuso Hospital \\
National Sapporo Hospital & Cancer Research Institute Hospital \\
Asahikawa Municipal Hospital & School of Medicine, Keio University \\
Sapporo Municipal Hospital & Tokyo Medical University \\
Nikko Memorial Hospital & Tokyo Women's Medical University School of Medicine \\
Aomori Prefectural Central Hospital & Tokyo Women's Medical University Daini Hospital \\
Akita University School of Medicine & Toranomon Hospital \\
Ota Nishinouchi Hospital & Japan Red Cross Hospital Medical Center \\
National Sendai Hospital & Mitsui Memorial Hospital \\
Sendai Municipal Hospital & Odawara Municipal Hospital \\
Fukushima Medical University & Kanagawa Cancer Center Hospital \\
Hoshi General Hospital & National Sagamihara Hospital \\
Yamagata University School of Medicine & Showa University Fujigaoka Hospital \\
Omiya Red Cross Hospital & Koseiren Takaoka Hospital \\
Ojiya General Hospital & Fukui Red Cross Hospital
\end{tabular}


Adjuvant UFT compared with CMF for node-positive breast cancer

Y Park et al

Appendix (Continued)

Kasukabe Municipal Hospital

Gunma University School of Medicine

Jichi Medical School

Saitama Social Insurance Hospital

Tochigi Cancer Center Hospital

Saiseikai Utsunomiya Hospital

Kameda General Hospital

Chiba Cancer Center Hospital

The Jikei University Kashiwa Hospital

Medical School, Nagoya City Hospital

Nagoya First Red Cross Hospital

Nagoya University School of Medicine

Yamada Red Cross Hospital

Kyoto Municipal Hospital

Kyoto University Faculty of Medicine

Kyoto Prefectural University of Medicine

Shiga Adult Disease Center

Osaka Medical College

Osaka Police Hospital

Osaka City University Medical School

Osaka University School of Medicine

Osaka Medical Center for Cancer and Cardiovascular Diseases

Kansai Medical University

Kinki University School of Medicine

National Osaka Medical Center

National Nara Hospital

Sakai Municipal Hospital

Toyonaka Municipal Hospital

Yao Municipal Hospital

Suita Municipal Hospital

Kansai Rosai Hospital

Hyogo Prefectural Amagasaki Hospital

Kobe Municipal Central Hospital

Kinki Central Hospital

Hyogo Medical Center for Adult Disease
National Kanazawa Hospital

Yaizu Municipal Hospital

Aichi Cancer Center Hospital

Kouseiren Kosei Hospital

lida Municipal Hospital

Gifu Municipal Hospital

Koseiren Gihoku Hospital

Yokkaichi Municipal Hospital

Shinshu University School of Medicine

Nagano Red Cross Hospital

Omoto Hospital

Kawasaki Medical School

Hiroshima Prefectural Hiroshima Hospital

National Iwakuni Hospital

National Kure Medical Center

Hiroshima Municipal Hiroshima Hospital

Hiroshima Municipal Asa Hospital

Research Institute for Radiation and Medicine, Hiroshima University

Anan Central Hospital for the Medical Association

Kochi Medical School

National Shikoku Cancer Center

Matsuyama Red Cross Hospital

School of Medicine, The University of Tokushima

Oita Prefectural Hospital

Kitakyushu Municipal Medical Center

Kyushu University Faculty of Medicine

National Kyushu Medical Center

National Miyakonojo Hospital

Saga Prefectural Koseikan Hospital

Ngasaki Municipal Hospital

Nagasaki Red Cross Hospital

School of Medicine Fukuoka University

Kumamoto Rosai Hospital 